\title{
A Circuit Design of High-precision Channel Equalizer for WSN-OFDM System
}

\author{
QU Xiao ${ }^{1, ~ a ~}$, XI Jincheng ${ }^{2, b}$, LIU Xinning ${ }^{3, c}$, YANG Jun ${ }^{4, d}$ \\ ${ }^{1,2,3}$ National ASIC System and Engineering Center, Southeast University, Nanjing 210096, China

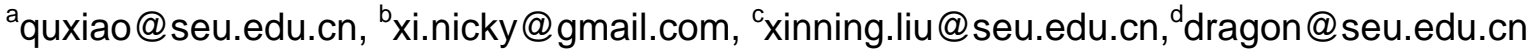

Key Words: WSN-OFDM; high-precision channel equalizer; fast LMMSE algorithm; circuit design

\begin{abstract}
Channel equalizer is a key component of WSN-OFDM (Wireless Sensor Networks Orthogonal Frequency Division Multiplexing) system. Traditional equalizer is mostly designed with LS algorithm, which comes with low precision in channel estimation. The previous LMMSE algorithm could reach a high accuracy, but it has high complexity and would consume high circuit resources as well. In order to improve precision of the channel equalizer with acceptable complexity, a fast LMMSE algorithm is proposed for channel estimation and equalization on the basis of LMMSE and DFT interpolation algorithm.The operation is simplified with the cyclic convolution properties and DFT properties.The FPGA verification results indicate that bits error ratio is only $2.3 \times 10^{-4}$ when signal-to-noise ratio is $16 \mathrm{~dB}$, which is $89.8 \%$ lower than that of traditional LS equalizer, at the expense of $27.27 \%$ more circuit resources.
\end{abstract}

\section{Introduction}

OFDM is an efficient multi-carrier modulation technique, which could achieve high data transmission rate and high spectral efficiency.Meanwhile, it could effectively restrain the interference of multipath and influence of the frequency-selective fading in the wireless channel [1,2]. Due to the time-varying characteristic of the wireless channel in WSN-OFDM system[3],channel estimation and equalization are needed for correct communication[4]. Traditional equalizer is mostly designed by LS(Least-Square) algorithm, which comes with low precision and low complexity[5,6]. Another common used algorithm is LMMSE (Linear Minimum-Mean-Square Error). The existing LMMSE algorithm could reach high accuracy, but comes with high complexity and consumes high circuit resources[7,8]. In order to improve the performance of signal transmission, a high-precision channel equalizer design with acceptable complexity is proposed.

\section{Channel Estimation and Equalization in OFDM}

Channel estimation and equalization are key techniques in OFDM system. Supposing that $x_{i, \mathrm{k}}$ is the sequence of transmitted data before inserting cyclic prefix, and $X_{i, k}$ is the frequency domain form, the received data is represented by $Y_{i, k}$, we have

$$
Y_{i, k}=X_{i, k} H_{i, k}+W_{i, k}
$$

where $H_{i, k}$ is the channel frequency response of the $k^{\text {th }}$ subcarrier in the $i^{\text {th }}$ OFDM symbol, and $W_{i, k}$ represents the white Gaussian noise.

Fig.1 indicates the algorithm architecture. The purpose of channel estimation is to estimate channel frequency response $\mathrm{H}_{i, k}$. And then channel equalization module calculates the transmitted data $\tilde{X}_{i, k}$ according to the received data $Y_{i, k}$ by using $\mathrm{H}_{i, k}$. Generally, LS has comparatively low complexity but low precision as well. LMMSE is superior to LS in precision, but comes with high complexity. Furthermore, because LMMSE needs a real-time estimation of the channel autocorrelation matrix $\mathrm{R}_{\mathrm{HpHp}}$ and signal-to-noise ratio (SNR), it's difficult for the circuit implementation of LMMSE. 


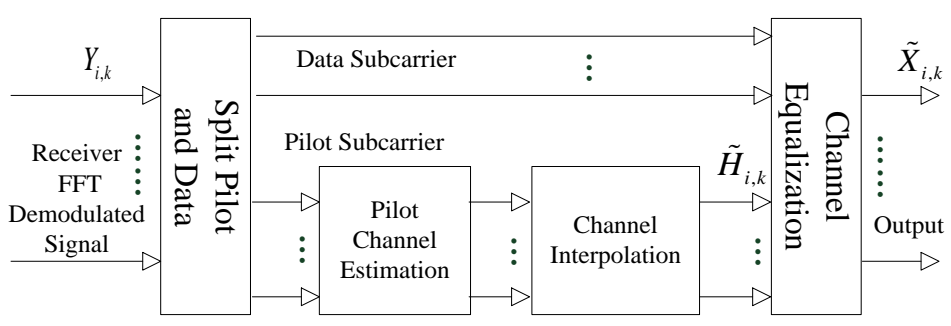

Fig.1 The architecture diagram of pilot assisted channel estimation and equalization algorithm in OFDM

\section{Algorithm Design}

\section{A. A Previous LMMSE Algorithm}

According to[8], an improved LMMSE algorithm has been proposed to solve the problem of real-time estimation of channel parameters. It firstly calculates $\mathrm{R}_{\mathrm{HpHp}}$ and SNR with the characteristics of the most significant paths.Then works out the estimates of channel frequency response in pilot subcarrier. It has also been proved that the coefficient matrix of LMMSE is a cyclic matrix. Finally,we get

$$
H_{p, l m m s e}(i)=R_{H_{p} H_{p}}\left(R_{H_{p} H_{p}}+\frac{\beta}{S N R} I\right)^{-1} H_{p, l s}(i)=\operatorname{CyclicMatrix}\left(\operatorname{IFFT}_{N_{p}}[P]\right) H_{p, l s}(i) \text {, }
$$

where, CyclicMatrix() means getting cyclic matrix from the first row. $H_{p, l s}(i)$ isLS channel frequency response, and

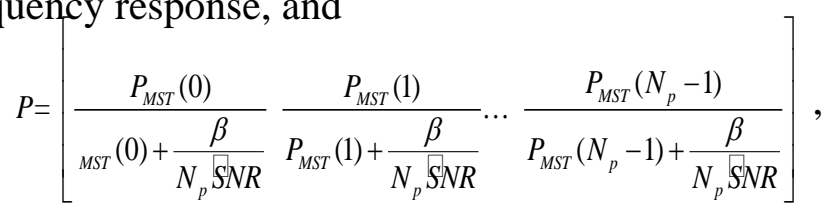

Which is calculated by power parameters $P_{\text {MST }}$, modulation factor $\beta$, estimated value of SNR and number of pilots $N_{p}$.

This algorithm is still too complicated for circuit design, since it contains matrix multiplication and many FFT/IFFT operations. Besides, the channel interpolation is not considered yet.

\section{B. Proposed Fast LMMSE Algorithm}

A fast LMMSE algorithm is proposed based on the combination of the previous LMMSE algorithm and DFT interpolation algorithm, as represented below.

Noted that Eq. 2 is a multiplication between a cyclic matrix and a column vector. This operation could be replaced by cyclic convolution. And transformation could be made due to the conjugate symmetry of DFT and the correlation properties between IFFT and FFT.

$$
\begin{aligned}
& \mathbb{H}_{p, l m m s e}(i)=\operatorname{CyclicMatrix}\left(\operatorname{IFFT}_{N_{p}}[P]\right) H_{p, l s}(i) \\
& =\left(I F F T\left[P^{*}\right]^{*}\right)^{T} \otimes H_{p, l s}(i) \\
& =\frac{1}{N} F F T[P]^{T} \otimes H_{p, l s}(i)
\end{aligned}
$$

Where, $*$ represents self-conjugate matrix, and $\otimes$ represents cyclic convolution operation.A simplified result could be obtained by cyclic convolution property $D F T\left[f_{1}(k) \otimes f_{2}(k)\right]=D F T\left[f_{1}(k)\right] \cdot D F T\left[f_{2}(k)\right]$ and double FFT operations $\operatorname{FFT}\{F F T[f(k)]\}=N f((N-k) \bmod N), k=0,1 \cdots(N-1)$, as shown below:

$$
\begin{aligned}
& H_{p, l m m s e}(i)=\operatorname{IFFT}\left\{\frac{1}{N_{p}} \operatorname{FFT}\left\{F F T[P]^{T}\right\} \cdot \operatorname{FFT}\left\{F F T\left[\tilde{h}_{p, l s}(i)\right]\right\}\right\} \\
& =N_{p} \operatorname{IFFT}\left\{\left[P\left(\left(N_{p}-k\right) \bmod N_{p}\right)\right]^{T} \cdot \tilde{h}_{p, l s}\left(i,\left(N_{p}-k\right) \bmod N_{p}\right)\right\}
\end{aligned}
$$

Comb-type pilots are evenly inserted into valid data for transmission. For the purpose of channel estimation, interpolation operation is need to obtain the estimates of channel frequency 
response in valid data subcarrier.

DFT interpolation algorithm is applied for channel interpolation [9].The number of pilots is $N_{p}$, and the number of data points between adjacent pilots is $N_{d}$, The DFT interpolation process could be divided into three steps:

1) $h_{p}(i)=\operatorname{IFFT}\left[H_{p, \text { lmmse }}(i)\right]$

2) $\tilde{h}(i)=\left[\tilde{h}_{p}(i, 0) \tilde{h}_{p}(i, 1) \ldots \tilde{h}_{p}\left(i, \frac{N_{p}}{2}-1\right) \ldots 0000 \ldots \tilde{h}_{p}\left(i, \frac{N_{p}}{2}\right) \tilde{h}_{p}\left(i, \frac{N_{p}}{2}+1\right) \ldots \tilde{h}_{p}\left(i, N_{p}-1\right)\right]$

3) $H(i)=F F T_{N_{d p}}[\tilde{h}(i)]$

Where, $N_{d p}=N_{p} *\left(1+N_{d}\right)$.

Then we could get channel interpolation results. And the channel frequency response of all valid data subcarriers has been estimated.

Considering the double IFFT operation IFFT $\{$ IFFT $[X]\}=\frac{1}{N}\left\{F F T\left\{I F F T^{*}[X]\right\}\right\}^{*}=\frac{1}{N} X((N-k) \bmod N)$, we substitute Eq. 5 into Eq. 6, and simplify it.

$$
h_{p}(i)=\operatorname{IFFT}\left[H_{p, \text { lmmse }}(i)\right]=P \cdot \tilde{h}_{p, l s}(i)
$$

It's easy to find that the fast algorithm is further simplified with the combination of improved LMMSE and DFT interpolation algorithm.Compared with the previous LMMSE, the fast LMMSE proposed reduces matrix multiplication and many FFT/IFFT operations. It only contains one IFFT, one FFT, one vector multiplication and some regular operation. The algorithm complexity mainly depends on the number of multiplication and division operations. And it can be worked out that the previous LMMSE in [8] needs $N_{p}{ }^{2}+\frac{3 N_{p}}{2} \log _{2}\left(N_{p}\right)+\frac{N_{d p}}{2} \log _{2}\left(N_{d p}\right)+2 N_{p}+2$ times of multiplication and division operations. While the proposed fast LMMSE needs $\frac{N_{p}}{2} \log _{2}\left(N_{p}\right)+\frac{N_{d p}}{2} \log _{2}\left(N_{d p}\right)+3 N_{p}+2$ times. When $N_{p}$ equals 8 and $N_{d p}$ equals 64 , the complexity of proposed fast LMMSE is $25.8 \%$ lower than the previous LMMSE.

\section{Circuit Design of Channel Equalizer}

\section{A. Pilot Architecture}

Pilot architecture of WSN-OFDM system is designed on the basis of fast LMMSE algorithm, as shown in Fig. 2.

\begin{tabular}{|c|c|c|c|c|c|c|c|c|c|c|c|c|c|c|c|c|c|c|}
\hline \multirow[b]{2}{*}{ sub-carrier: } & \multicolumn{11}{|c|}{ d: data } & \multicolumn{2}{|c|}{ p: pilot } & \multicolumn{5}{|c|}{ v: virtual sub-carrier } \\
\hline & $\mathbf{v}$ & 3d & $\mathbf{p}$ & 7d & $\mathbf{p}$ & 7d & $\mathbf{p}$ & $7 d$ & $\mathbf{p}$ & $7 v$ & $\mathbf{p}$ & 7d & $\mathbf{p}$ & $7 d$ & $\mathbf{p}$ & 7d & $\mathbf{p}$ & $3 d$ \\
\hline IFFT order: & $\mathbf{0}$ & & 4 & & 12 & & 20 & & 28 & & 36 & & 44 & & 52 & & 60 & \\
\hline $\begin{array}{l}\text { Equalization } \\
\text { order: }\end{array}$ & 28 & & 31 & & 39 & & 47 & & 55 & & 0 & & 8 & & 16 & & 24 & \\
\hline
\end{tabular}

Fig. 2Pilot architecture of WSN-OFDM system

The 64-point IFFT/FFT architecture is chosen for modulation and demodulation in WSN-OFDM system, with consideration of data transmission rates and band width.Under the architecture, there are 48 valid data subcarriers for data transmission,8 pilot subcarriers for channel estimation and 8 virtual subcarriers. The virtual subcarriers distribute in DC position and the middle part of the spectrum. In this way, DC component of the signal would be removed for power saving, and peak-to-average power ratio could be reduced effectively. 


\section{B. Framework and Parameters of Channel Equalizer}

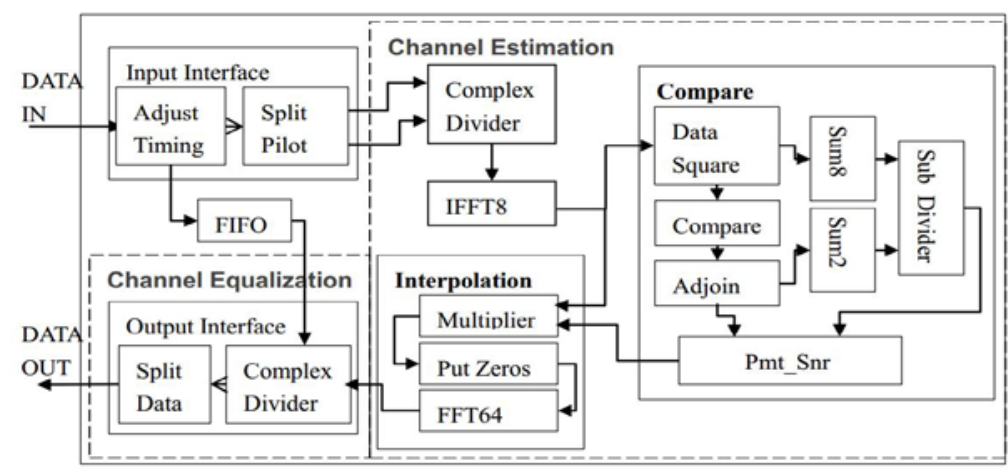

Fig. 3Framework of channel equalizer design

A simplified 20-bit floating point format is customized for circuit design, which not only retains the advantages of floating point data,like no overflowing and high-precision for small value[10,11], but also reduces the circuit complexity.There are 1 sign bit, 5 exponent bits and 14 fraction bits in the data format.

According to fast LMMSE algorithm and pilot architecture, framework of channel equalizer in WSN-OFDM is designed as shown in Fig. 3. The channel estimation module and the channel equalization module are two main components.

With consideration of the requirements of WSN-OFDM system, parameters of the channel equalizer are given in Table 1:

Table 1Parameters of channel equalizer

\begin{tabular}{|c|c|c|c|}
\hline Parameters & Value & Parameters & Value \\
\hline System clock & $20[\mathrm{MHz}]$ & Data rate & $48[\mathrm{Mbps}]$ \\
\hline Modulate mode & $16-\mathrm{QAM}$ & Pilot subcarriers & 8 \\
\hline Data subcarriers & 56 & OFDMsymbol length & $4[\mathrm{us}]$ \\
\hline Signal bandwidth & $17.5[\mathrm{MHz}]$ & Guard interval length & $0.8[\mathrm{us}]$ \\
\hline Channel bandwidth & $20[\mathrm{MHz}]$ & Sub-carrier interval & $312.5[\mathrm{kHz}]$ \\
\hline
\end{tabular}

\section{Verification and Analysis}

\section{A. Circuit Simulation}

In order to verify the function of the design,the co-simulation of Modelsim and Matlab is performed. The results are compared with LS algorithm.

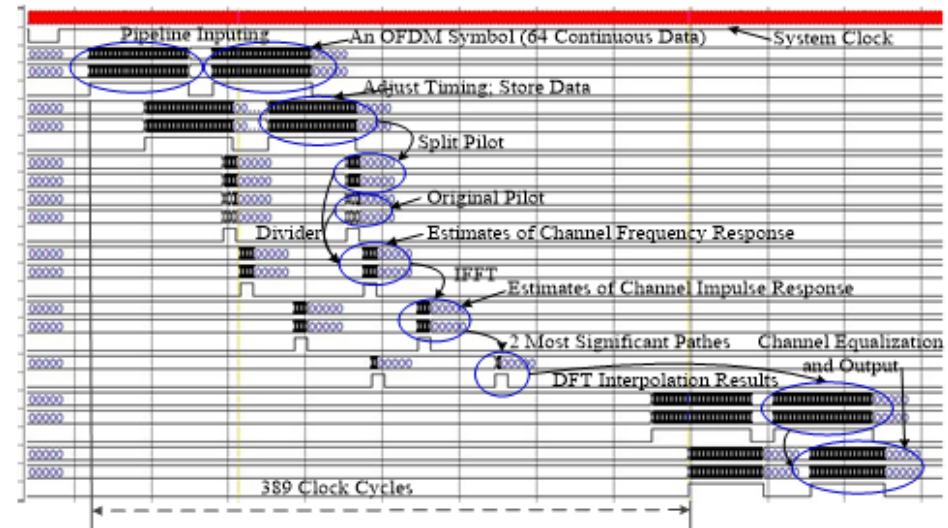

Fig. 4The timing simulation diagram of designed equalizer circuit

In Modelsim simulation, two OFDM symbols with 16 cycles' interval have been put into the designed equalizer circuit. And the timing simulation diagram is shown in Fig. 4.

According to Fig. 4, we get that 1)the designed equalizer circuit runs correctly as the proposed fast LMMSE algorithm; 2)pipeline design meets the requirement of continuous signal processing in 
WSN-OFDM system; 3) the total time-delay of equalizer circuit is 389 clock cycles (19.45us), which meets the requirement of real time communication.

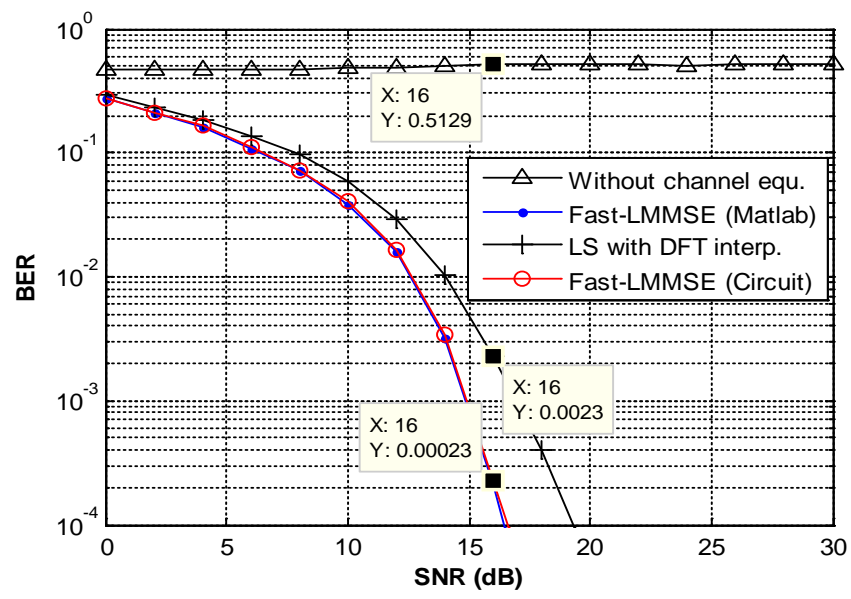

Fig. 5System simulation results: BER-SNR curves

Fig. 5 shows the system simulation results. The precision of channel equalizer is evaluated by bits error ratio(BER) in different SNR. It could be concluded that 1)the proposed fast LMMSE is superior to LS with DFT interpolation; 2) the curve of fast LMMSE in designed circuit matches the curve of fast LMMSE in Matlab very well, which indicates the circuit runs correctly; 3)the BER of designed equalizer is only $2.3 \times 10^{-4}$ when SNR is $16 \mathrm{~dB}$, which is $89.8 \%$ lower than that of traditional

\section{B. Analysis of Circuit Resources Consumption}

LS equalizer.

The hardware design of equalizer is compiled and synthesized in Quartus II software. The FPGA chip used for verification is EP3SL340H1152C2 from Altera.The circuit resources consumption of the fast LMMSE equalizer and traditional LS with DFT interpolation equalizer are shown in Table 2.

Table 2Circuit resources consumption of equalizer

\begin{tabular}{cccc}
\hline Equalizer type & ALUTs & Registers & Logic utilization \\
\hline Fast LMMSE & $21225 / 270400$ & $27316 / 270400$ & $14 \%$ \\
LS & $16967 / 270400$ & $23163 / 270400$ & $11 \%$ \\
\hline
\end{tabular}

The fast LMMSE design occupies about $14 \%$ of the total logic resources in EP3SL340H1152C2 chip. Combining with the above analyses, compared with traditional LS equalizer, the BER of designed fast LMMSE equalizer is only $2.3 \times 10^{-4}$ when SNR is $16 \mathrm{~dB}$, which is $89.8 \%$ lower than that of traditional LS equalizer, with only $27.27 \%$ more circuit resources. The high-precision channel equalizer has been proved to work well.

\section{Conclusions}

This paper concentrates on the algorithm optimization and circuit design of high-precision channel equalizer. To be specific, 1) a fast LMMSE algorithm is proposed for channel estimation and equalization, the complexity of which is 25.8\% lower than previous LMMSE algorithm; 2)the framework of channel equalizeris designed and a simplified 20-bit floating point format is customized for circuit design, which leads to the implement of high-precision channel equalizer.

The verification results of FPGA and Matlab platform indicate that BER of designed channel equalizer is only $2.3 \times 10^{-4}$ when SNR is $16 \mathrm{~dB}$, which is $89.8 \%$ lower than that of traditional LS equalizer, with only $27.27 \%$ more circuit resources.

\section{References}

[1] Jeon W G, Chang K H, Cho Y S. An equalization technique for orthogonal frequency-division multiplexing systems in time-variant multipath channels [J]. Communications, IEEE Transactions 
on, 1999, 47(1): 27-32.

[2] Li Y G, Stuber G L. Orthogonal frequency division multiplexing for wireless communications [M]. Springer, 2006.

[3] Senol H, Panayirci E, Poor H V. Joint channel estimation and equalization for OFDM based broadband communications in rapidly varying mobile channels[C]//GLOBECOM Workshops (GC Wkshps), 2010 IEEE. IEEE, 2010: 736-740.

[4] Jeon W G, Chang K H, Cho Y S. An equalization technique for orthogonal frequency-division multiplexing systems in time-variant multipath channels [J]. Communications, IEEE Transactions on, 1999, 47(1): 27-32.

[5] Chunlong $\mathrm{H}, \mathrm{Li} \mathrm{H}$. Pilot-aided channel estimation techniques in OFDM system[C]//Communication Software and Networks, 2009. ICCSN'09. International Conference on. IEEE, 2009: 143-146.

[6] Lim C H, Han D S. Robust LS channel estimation with phase rotation for single frequency network in OFDM[J]. Consumer Electronics, IEEE Transactions on, 2006, 52(4): 1173-1178.

[7] Noh M, Lee Y, Park H. Low complexity LMMSE channel estimation for OFDM[C]//Communications, IEE Proceedings-. IET, 2006, 153(5): 645-650.

[8] Zhou W. A study of channel estimation for OFDM systems and system capacity forMIMO-OFDM systems[J]. University of Hong Kong. HongKong, 2010: pp 65-68.

[9] Li D, Guo F, Li G, et al. Enhanced DFT interpolation-based channel estimation for OFDM systems with virtual subcarriers[C]//Vehicular Technology Conference, 2006. VTC 2006-Spring. IEEE 63rd. IEEE, 2006, 4: 1580-1584.

[10] ANSI/IEEE 754-1985. American National Standard - IEEE Standard for Binary Floating-Point Arithmetic. American National Standards Institute, Inc., New York, 1985.

[11] IEEE 754-2008. IEEE Standard for Floating-Point Arithmetic. IEEE Computer Society. August 2008. 